Proceedings of SALT 26: 754-774, 2016

\title{
Presupposition projection from the scope of none: Universal, existential, or both? *
}

\author{
Jérémy Zehr \\ University of Pennsylvania \\ Lyn Tieu \\ Macquarie University
}

\author{
Cory Bill \\ Macquarie University \\ Jacopo Romoli \\ Ulster University
}

\author{
Florian Schwarz \\ University of Pennsylvania
}

\begin{abstract}
The nature of presupposition projection from the scope of quantificational expressions, particularly negative ones, continues to be both theoretically and empirically controversial. We experimentally investigate the interpretation of sentences like None of the bears won the race, which could in principle be associated with three projection options, giving rise to: an existential reading, on which at least one of the bears participated and none won; a universal reading, on which all of the bears participated and none won; and a presuppositionless reading on which none of the bears both participated and won. We used a covered box task to investigate English-speaking adults' and children's interpretations of such sentences. The results from the adult participants provide empirical evidence for all three readings; the results from the child participants, however, reveal no evidence for the existential reading. We discuss our results in light of competing theoretical accounts, focusing on options for reconciling the theories with the experimental data from both adults and children.
\end{abstract}

Keywords: presupposition, presupposition projection, quantification, experimental pragmatics, covered box, truth value judgment task

* For helpful comments and suggestions, we would like to thank Emmanuel Chemla, Stephen Crain, Alexandre Cremers, Danny Fox, Matt Mandelkern, Clemens Mayr, Philippe Schlenker, Jesse Snedeker, Yasu Sudo, and audiences at SALT26, the Amsterdam Colloquium 2015, Utrecht, and the DGfS workshop on Presuppositions in Language Acquisition. Work on this project was supported by NSF grant BCS-1349009 to Florian Schwarz, by the European Research Council under the European Union's Seventh Framework Programme (FP/2007-2013) / ERC Grant Agreement n.313610, ANR-10-IDEX-0001-02 PSL*, and ANR-10-LABX-0087 IEC, and by the Alexander von Humboldt-Stiftung and the Andrew W. Mellon Foundation as part of the SIAS Summer Institute 'The Investigation of Linguistic Meaning.'

(C)2016 Zehr, Bill, Tieu, Romoli and Schwarz 
Presupposition projection from the scope of none

\section{Introduction}

Presupposition projection plays a central role in presupposition theory, and serves as a key diagnostic for identifying presuppositional expressions. For example, the sentence in (1a) gives rise to the inference in (1b), which is standardly analyzed as a presupposition in virtue of its projection out of linguistic environments like negation (2a), questions (2b), and the scope of modals (2c). ${ }^{1}$

(1) a. Bear won the race.

b. $\rightsquigarrow$ Bear participated in the race

(2) a. Bear didn't win the race.

b. Did Bear win the race?

c. It is possible that Bear won the race.

While a particular projection pattern is almost universally accepted as a key diagnostic to identify presuppositions, much remains unknown about the specific mechanisms that underlie projection behavior. In particular, a central challenge lies in identifying which properties of presuppositional expressions and/or linguistic environments are actually responsible for projection effects.

Moreover, while there is a fairly broad consensus with regards to the empirical facts concerning sentences like $(2 \mathrm{a}-2 \mathrm{c})$, projection in quantificational sentences like (3) is a subject of ongoing controversy. Theories differ in whether they associate (3) with existential projection of the presupposition (i.e. at least one of the bears participated), universal projection (i.e. all of the bears participated), or both, with each side claiming empirical adequacy.

(3) None of the bears won the race.

The picture is further complicated by the fact that the projected inference does not always seem to be present. Negative environments in particular are known to allow for presuppostion suspension: both the simple negative sentence (2a) and the negatively quantified sentence (3) can consistently be followed by a statement that is incompatible with the presupposition in question, as illustrated in (4a) and (4b).

a. Bear didn't win the race - he didn't even participate!

b. None of the bears won the race - none of them even participated!

1 The influential view of presuppositions advanced by Stalnaker 1970, 1974; Karttunen 1974; Heim 1983 sees them as admittance conditions on the assertion of the relevant sentence. We use the term 'inference' broadly here to include various aspects of meaning associated with a sentence, including potential cases of (global) accommodation; see Chemla 2009a for discussion. 
So in fact, a sentence like (3) could in principle be associated with three distinct readings, paraphraseable as in $(5 a-5 c)$.

None of the bears won the race.

a. EXISTENTIAL: At least one of the bears participated and no bear won.

b. UNIVERSAL: All of the bears participated and no bear won.

c. PResupPositionless: None of the bears both participated and won.

It is important at this stage to distinguish between predicted readings of a sentence and the precise nature of the projected presupposition underlying the readings. This is because, as we discuss below in further detail, theories can predict the different readings either directly via presupposition projection or via the predicted presupposition in combination with the effect of some other mechanism. For instance, the EXISTENTIAL reading in (5a) could be the result of existential projection of the presupposition of win directly, or it could arise through a kind of weakening (e.g., through domain restriction) of a universal projection reading. Conversely, a UNIVERSAL reading could be associated with universal projection directly, or derived through the strengthening of an existentially projected presupposition.

We experimentally investigate projection from the scope of negative quantifiers, focusing on three main research questions. First, we aim to establish the empirical facts regarding the availability of each of the three readings in question. Second, we aim to investigate the relationship between the available readings, for theories of projection differ on whether and how the EXISTENTIAL vs. UNIVERSAL readings are derived from one another. Third, we aim to investigate the status of the PRESUPPOSITIONLESS reading, examining in particular the question of whether this reading might correspond to a sentence's basic meaning, or instead to a derived meaning.

To address these questions, we investigated the interpretation of quantificational sentences like (5) in English-speaking adults and 4-6-year-old children. The main results are as follows. In relation to the first question, the adult data provide evidence for the existence of all three of the readings. The child data, on the other hand, provide no evidence for the EXISTENTIAL reading. As we will discuss in relation to the second research question, on certain assumptions about the acquisition of basic and derived meanings, this finding may suggest that the UNIVERSAL reading is basic while the EXISTENTIAL reading is derived. Finally, in relation to the third research question, our results present a more complicated picture. On the one hand, reaction time data from the adult participants reveal delays for the PRESUPPOSITIONLESS reading, relative to the EXISTENTIAL and UNIVERSAL readings. This finding suggests that PRESUPPOSITIONLESS readings are not basic, but rather involve other processes, consistent with previous findings in the literature (Chemla \& Bott 2013; Romoli \& Schwarz 2014). On the other hand, children appear to access 
the PRESUPPOSITIONLESS reading with ease, which is surprising in light of some previous developmental results. We suggest that children may be accessing this reading differently from adults. We discuss how our results constrain the theoretical options for deriving the three readings in (5), as well as the link between these theoretical options and children's acquisition of the various readings.

The remainder of the paper is organized as follows. Section 2 provides a brief discussion of how existing theories can account for the three readings in question, and reviews pertinent experimental studies in the literature. Section 3 presents the main experiments, the findings of which we discuss more generally in Section 4. Section 5 concludes the paper.

\section{Background}

\subsection{Theoretical landscape}

Theories of presupposition projection in quantificational sentences can be roughly grouped into three camps: theories that only predict universal projection, theories that only predict existential projection, and theories that allow for both projection options. We only consider theories that predict existential and universal readings directly via presupposition projection mechanisms as belonging to the last category. The other two approaches derive only one of the two readings through such mechanisms, but can appeal to additional mechanisms to derive the respective other reading. In grouping theories, we focus in particular on their predictions with respect to negative quantificational sentences and we ignore here three additional dimensions of variation discussed in the literature: variation with respect to the quantifier involved in the sentence (see Chemla 2009b, 2010; George 2008; Fox 2011; Sudo 2012; Mandelkern 2016; Mayr \& Sauerland 2016, among others), variation with respect to the trigger involved (see Charlow 2009; Fox 2011; Sudo 2012), and inter-speaker variation with respect to the available readings (see Sudo, Romoli, Fox \& Hackl 2012).

Universal-only theories The first group of theories predicts only universal presupposition projection for sentences like (5) (Heim 1983, Schlenker 2008, 2010; George 2008; Fox 2012; Chemla \& Schlenker 2012; Mayr \& Sauerland 2016). ${ }^{2}$ These theories account for the UNIVERSAL reading of (5) directly via presupposition

2 To be precise, trivalent theories such as George 2008; Fox 2008, 2012 and Sudo et al. 2012 make more nuanced predictions than 'pure' Universal-Only theories, by varying the force of projection with the quantifier. Moreover, the projected presupposition predicted by such theories is the disjunctive one in (i), which is weaker than the UNIVERSAL but stronger than the EXISTENTIAL reading. When the assertion is true, however, as was always the case in the study we will describe (i.e. the winner was not a bear), (i) becomes equivalent to the UNIVERSAL reading, All of the bears participated, so we group them together in the present discussion. 
projection. While they do not predict genuine EXISTENTIAL readings, these theories can account for them by appealing to other mechanisms, such as domain restriction. That is, while assuming that (6), repeated from above, always projects the universal presupposition in (7), they can capture the EXISTENTIAL reading via a contextual restriction on the set of bears to those who satisfy the presupposition (i.e. None of the bears [who participated in the race] won the race).

(6) None of the bears won the race.

(7) All of the bears participated in the race.

Existential-only theories Existential-only theories of presupposition projection only derive an EXISTENTIAL reading directly via the existential presupposition in (8) (Beaver 1994; van der Sandt 1992; Geurts 1998; Mandelkern 2016). However, similarly to Universal-Only theories, these accounts also have the option of deriving the other reading through independent mechanisms, e.g., via a separate strengthening mechanism. To our knowledge, there are no detailed proposals as to what this mechanism would be, but a possible option would be a preference for homogeneity across domains, which would strengthen the EXISTENTIAL reading to a UNIVERSAL one. $^{3}$

At least one of the bears participated.

Universal + existential projection A third theoretical option predicts both EXISTENTIAL and UNIVERSAL readings directly, depending on how the presupposition projection mechanism interacts with the different quantifiers (Chierchia 1995, 2010). Scalar implicature-based theories of presupposition, such as Chemla 2010 and Romoli 2012, 2014 also predict both readings. ${ }^{4}$

As a final note, all theories under consideration assume a presupposition suspension mechanism of some sort (e.g., local accommodation), which handles cases where projection is not observed, i.e. cases that involve what we refer to as a PRESUPPOSITIONLESS reading.

i. Either all of the bears participated and no bear won, or at least one bear participated and won.

3 Mandelkern (2016) sketches a direction to explore in implementing this strengthening mechanism based on decomposing the negative quantifiers none as not some and a homogeneity presupposition associated with the embedded some.

4 On this approach, the predicted universal or existential inference depends on the alternatives that the presupposition or implicature is computed upon, i.e. whether they include among the alternatives only none of the bears participated or also not all of the bears participated. 
Presupposition projection from the scope of none

In sum, existing theories in the literature can be divided roughly into three camps. All of them can derive the three readings either directly or via an extra mechanism. Against this theoretical background, our study has three main goals. First, we will assess whether all three readings are available for sentences like (5). Second, if both EXISTENTIAL and UNIVERSAL readings are available, we will explore the relationship between them, in particular investigating whether one may be derived from the other. Finally, we will investigate the nature of the PRESUPPOSITIONLESS reading, in particular whether it corresponds to a literal meaning of sentences like (5) or rather involves further processes (Chemla \& Bott 2013; Romoli \& Schwarz 2014).

\subsection{Previous experimental studies}

In this section, we review three studies on presupposition projection in quantificational sentences, paying special attention to the case of negative quantifiers. The results of these studies provide evidence for UNIVERSAL readings as well as for non-universal readings. Crucially, however, they do not allow us to distinguish between PRESUPPOSITIONLESS and EXISTENTIAL readings. We will then explain how our experimental design allows for the detection of all three readings in $(5 a-5 c)$.

The first experimental investigation of presupposition projection from the scope of quantifiers that we are aware of is Chemla (2009a), who used an inferential task. The experimental conditions relevant to our discussion here involved presenting participants with negatively quantified sentences containing a presupposition trigger (9a) or scalar expression (10a). Participants were asked directly whether such sentences suggested that the corresponding positive, universally quantified inference was warranted. Chemla (2009a) found that participants endorsed universal presuppositional inferences like (9b) significantly more often than universal scalar inferences like (10b) (respectively over $80 \%$ vs. less than $30 \%$ ). These results suggest that sentences like (9a) are indeed associated with a UNIVERSAL reading (and to a higher degree than sentences like (10a)).

(9) a. None of these 10 students knows that he is lucky.

b. $\rightsquigarrow$ Each of these 10 students is lucky

(10) a. None of these 10 students missed all of their exams.

b. $\rightsquigarrow$ Each of these 10 students missed some of their exams

Evidence for non-universal readings comes from two truth value judgment studies. Sudo et al. (2012) presented participants with pictures such as the one in Figure 1 and asked whether such a picture was a good match for the accompanying negatively quantified presuppositional sentence. Crucially, the candidate UNIVERSAL 
Zehr, Bill, Tieu, Romoli and Schwarz

None of these three circles have the same color as both of the squares in their own cell

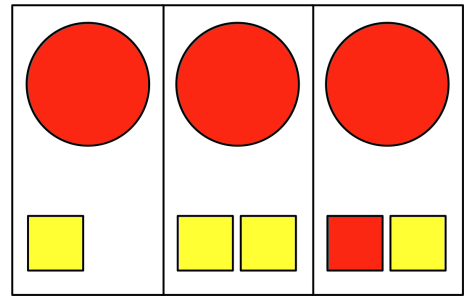

Figure 1 Target trial from Sudo et al. (2012)

No circle has the same color as the square to which it is connected

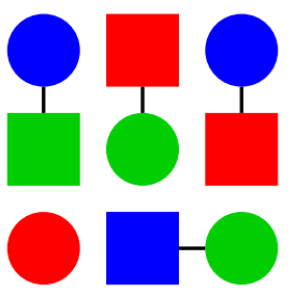

Figure 2 Target trial from Geurts \& van Tiel (2015)

reading of the sentence (i.e. that each of the three circles has exactly two squares in its own cell) was not compatible with the picture in the critical condition. Given the presuppositional trigger (both of the squares in their own cell), the candidate UNIVERSAL reading of the sentence requires each cell to contain two squares, but there is only one square in the left-most cell in Figure 1. Nonetheless, more than half of the participants responded that the picture was a good match for the sentence in this condition, indicating the availability of an alternative, non-universal reading. In a series of truth value judgment tasks using a similar set-up, Geurts \& van Tiel (2015) found up to $92 \%$ acceptance for negatively quantified presuppositional descriptions of pictures such as Figure 2, where the bottom left circle is not connected to any square. Given the presuppositional trigger (the square to which it is connected), the picture is incompatible with the candidate UNIVERSAL reading of the sentence, according to which each circle is connected to a square.

While these experiments provide evidence for at least two types of readings, they do not establish the existence of an independent EXISTENTIAL reading per se. The responses that the participants gave in Sudo et al. (2012) and Geurts \& van Tiel (2015) are compatible both with EXISTENTIAL and PRESUPPOSITIONLESS readings. 
Presupposition projection from the scope of none

The experiments we present in the next section were designed to investigate the existence and availability of all three of the readings in question (UNIVERSAL, EXISTENTIAL, and PRESUPPOSITIONLESS). To detect for these three readings in principle, the design involved various pairwise comparisons across conditions that together would provide direct evidence for the existence of a given reading. In contrast with the previous experiments, our design could differentiate between the EXISTENTIAL and the UNIVERSAL reading on the one hand, and the EXISTENTIAL and the PRESUPPOSITIONLESS reading, on the other.

\section{Experiments}

\subsection{Experiment 1}

Methods and design We used a variant of the Covered Box paradigm (Huang, Spelke \& Snedeker 2013). Each trial began with a context screen depicting a group of animals; four of these animals were taking part in a race, while another three animals, who were clearly not taking part in the race, were depicted inside of a house separate from the racetrack. A context sentence was presented auditorily along with this context picture (see Figure 3 for illustration). After the playback of the context sentence ended, participants were invited to press a key to reveal the test screen (Figure 4), which was about what the animals did later in the day, during an afternoon race. On this screen, there were two pictures instead of one, with one represented as hidden from view (see Figure 4). The participants were instructed that only one of the two pictures would correspond to the description provided by the sentence, and their job was to determine which one. Note that because one of the pictures was hidden, participants could freely imagine the situation depicted. If they thought the visible picture matched the test sentence, they could report it as a good match (by pressing $\mathbf{F}$ on their keyboard), but if they thought the visible picture did not correspond to the test sentence, they could freely assume that the situation depicted in the covered picture was a better match in whatever concrete way their interpretation of the sentence required (in this case, they would press $\mathbf{J}$ on their keyboard). ${ }^{5}$

The crucial manipulation between conditions involved varying how the visible picture related to the three readings under investigation. In the critical OnlySome condition (see Figure 4), the visible picture on the screen showed only a subset of the mentioned animals participating in the race, making it consistent with the EXISTIENTIAL and PRESUPPOSITIONLESS readings but not with the UNIVERSAL reading. In the critical NoRunner condition (see Figure 5), the visible picture showed

5 Note that the picture that was 'covered' by the black box was never revealed to participants. This was done to avoid biasing participants towards a particular interpretation of the test sentence. 
Zehr, Bill, Tieu, Romoli and Schwarz

[ During the morning race, these three bears did really well, and in the end, one of them won. I thought they would do the same later in the day as well, but... I (audio)

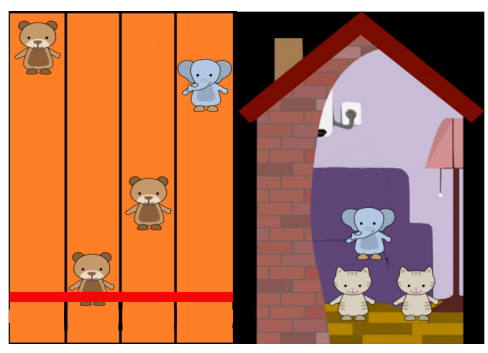

Press any key to continue.

Figure 3 Context screen for a test trial.

none of the mentioned animals participating in the race, making it consistent only with the PRESUPPOSITIONLESS reading. The experiment also included TrueControl items where the visible picture depicted all the mentioned animals participating in the race but none of them winning (making it a good match under any reading), and FalseControl items minimally differing from TrueControl items in that one of the mentioned animals in the visible picture did win the race (making it inconsistent with all of the relevant readings). Participants saw four repetitions of the OnlySome and of the NoRunner conditions (with different animals under discussion each time), and two repetitions each of the TrueControl and FalseControl conditions, for a total of 12 test trials. In addition, participants saw eight filler items, half corresponding to "covered" targets and half to "visible" targets. These were meant to assess participants' understanding of the task; for example, four of the fillers tested participants' ability to recognize which animals had participated in the race and which hadn't. ${ }^{6}$

The experimental trials were presented in three blocks: the first two blocks each included a TrueControl item, a FalseControl item, a "visible" filler, and a "covered" filler, along with either four critical OnlySome targets or four NoRunner targets (with order varied between groups). The last block consisted exclusively of the four "participate" filler trials. The order of the items was pseudorandomized to ensure an even distribution of "visible", "covered", and critical targets in each blocksequence. There were a total of four possible orders, derived from crossing block order (OnlySome-first vs. NoRunner-first) with item order (Normal vs. Reversed).

6 Since these items explicitly tested for the phrase participate in the race, which is a direct paraphrase of the presupposition at play, we presented them last to prevent any undue influence on critical trials. 
[ None of the bears won the afternoon race. ] (audio)
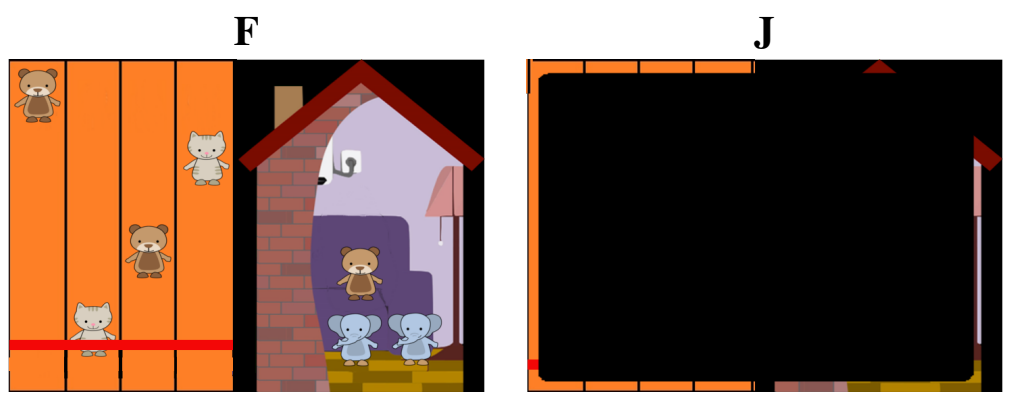

Figure 4 Test screen for an OnlySome trial.

Participants \& procedure Forty-eight adult participants were recruited via Amazon Mechanical Turk, and redirected to the Ibex server where the experiment was implemented and hosted (an archived version can be found at http://spellout.net/ ibexexps/SchwarzLabArchive/NoWin/experiment.html). The experiment took on average less than 10 minutes to complete, and participants received $\$ 1$ for their participation.

At the beginning of the experiment, participants saw an instruction screen explaining the task and introducing the different animals. Before responding to actual experimental items, participants first saw two practice trials about animals taking part (or not taking part) in morning and afternoon races; these trials were meant to familiarize participants with the task, and did not contain critical words like win or participate. We recorded both responses (i.e. picture selections of visible vs. covered) and response times.

Predictions The primary goal of this experiment was to settle the empirical issue of which of the readings in (5) are available for presuppositional expressions in the scope of none. Each of the three readings would be associated with a unique response pattern across the target conditions, as summarized in Figure 5. The transition in a given row from $\checkmark$ to $\times$ corresponds to potential contrasts that would uniquely support the availability of the reading in question: the UNIVERSAL reading predicts a contrast between the TrueControl (visible) and OnlySome (covered) conditions; the EXISTENTIAL reading predicts a contrast between the OnlySome (visible) and NoRunner (covered) conditions; and the PRESUPPOSITIONLESS reading predicts a contrast between the NoRunner (visible) and FalseControl (covered) conditions. Note that if all three readings were available with full generality and there were no differences between them, we might face ceiling effects in the form of across- 


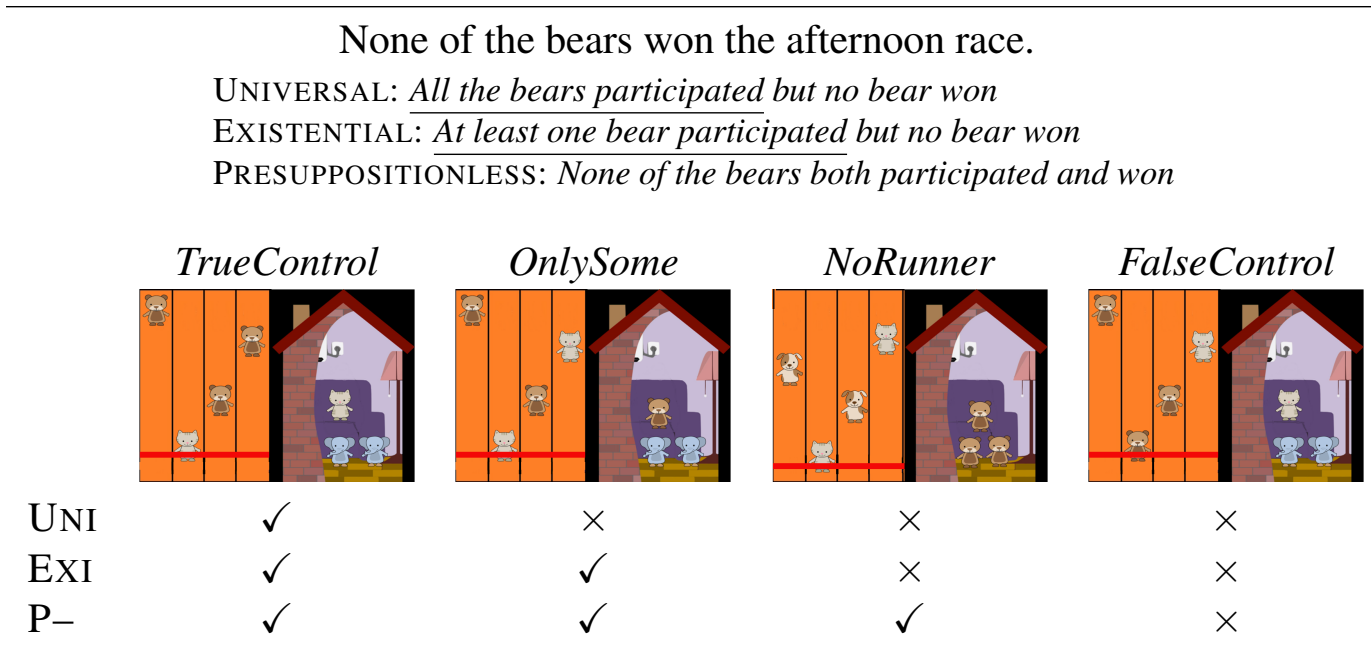

Figure 5 Predictions of each reading in the test conditions. $\checkmark$ indicates a visible picture selection; $\times$ indicates a covered picture selection.

the-board acceptance (setting aside the FalseControl condition). However, it is plausible that the relative availability of the readings in question might differ, which would open up some room to detect differences. Alternatively, if speakers randomly sampled from the available readings, we might also expect statistical differences to emerge in the acceptance patterns. What is crucial in our design is that any positive evidence of differences between two conditions can be taken to support the existence of the relevant reading.

Results Across all the analyses, we collapsed the control and filler trials, and from this point on we will refer to them more generally as the control trials/conditions. Six of the 48 participants were excluded from analyses because they scored less than $75 \%$ accuracy on these control trials. Figure 6 plots the rates of covered picture choices by condition for the remaining 42 participants.

We ran mixed-effect logistic regression analyses on the responses of the 42 adult participants. The results were descriptively similar in each of the four groups, and including block and item order as random effects did not qualitatively change the statistical patterns. We used the glmer function from the lme4 package (version 1.1.7) for $\mathrm{R}$ (version 3.1.2) to compute the most complex random-effect models that would converge, following Barr, Levy, Scheepers \& Tily (2013). Our models predicted the observation of covered responses depending on Condition with random intercepts for Participant and Trial, and no random slopes. Each condition appeared to differ significantly from one another. In particular, responses to TrueControl 


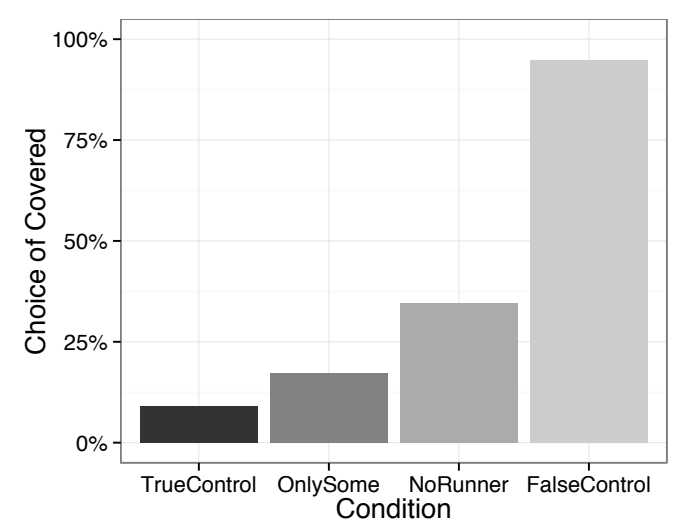

Figure 6 Mean percentage of covered picture selections in each condition. The TrueControl and FalseControl bars include the responses to both the control and the filler trials.

differed from those to OnlySome $(p<.05)$, responses to OnlySome differed from those to NoRunner $(p<.001)$, and responses to NoRunner differed from those to FalseControl $(p<.001)$.

Figure 7 plots the response times for each choice (visible vs. covered) by condition. Trials with RTs greater than two standard deviations above the mean were removed from the data (constituting $3.6 \%$ of the data, with equal distribution across NoRunner and OnlySome conditions).

A mixed-effect regression analysis, with random intercepts for participants and items, revealed that visible picture selections in the NoRunner condition took significantly longer than in the OnlySome condition $(M=4140 \mathrm{~ms}$ vs. $M=3946 \mathrm{~ms}$; $\beta=-457.6, S E=209.6, t=-2.184)$.

Discussion Our experiment set out to investigate the availability of the EXISTENTIAL, UNIVERSAL, and PRESUPPOSITIONLESS readings of sentences like (11).

(11) None of the bears won the race.

Recall that previous empirical work had reported evidence of both a UNIVERSAL and a non-universal reading of such sentences. However, the existing data did not allow us to determine whether the non-universal readings corresponded to EXISTENTIAL readings or to PRESUPPOSITIONLESS readings. Our experiment included conditions which allowed us to detect whether participants were accessing one or both of these readings, in addition to the UNIVERSAL reading. In particular, 


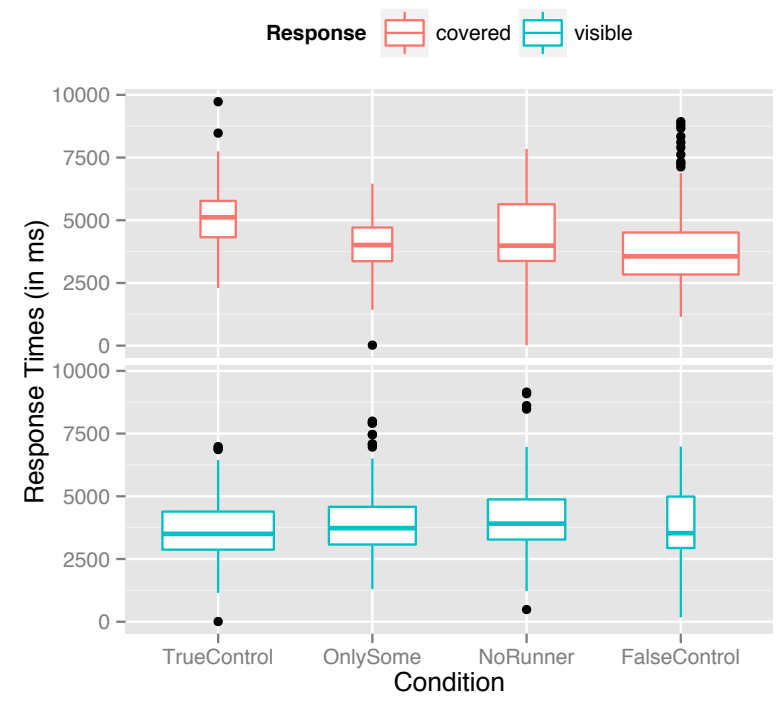

Figure 7 Response times for covered (top) and visible (bottom) choices in each condition. Larger boxes indicate more data points.

for the UNIVERSAL reading this meant looking for a contrast in the rate of covered picture selections between the TrueControl and OnlySome conditions. Our results indeed indicate the presence of such a contrast, providing evidence that this reading was accessed by the participants we tested. Similarly, we find evidence for the EXISTENTIAL and PRESUPPOSITIONLESS readings by comparing the OnlySome and NoRunner, and NoRunner and FalseControl conditions, respectively. In sum, we found experimental evidence for all three readings of (11). We can therefore provide a positive answer to the first question we raised in the Introduction, namely that all three readings under question are indeed possible.

The next issue we turn to is how the various readings relate to one another. The response time results provide a first insight, as we found slower response times for visible picture selections in the NoRunner condition, compared to the OnlySome condition. Choosing the visible picture in the NoRunner condition corresponded to accessing a PRESUPPOSITIONLESS reading. Selection of the visible picture in the OnlySome condition could be due to participants accessing the PRESUPPOSITIONLESS or the EXISTENTIAL reading. Given the contrast in response times, the results suggest that responding based on the PRESUPPOSITIONLESS reading is associated with a slow-down. One way to interpret this, which speaks to our third research question, is that the observed slow-down is indicative of an additional step that is 
Presupposition projection from the scope of none

required in order to access the PRESUPPOSITIONLESS reading. Such an interpretation is in line with previous results in the literature (Chemla \& Bott 2013; Romoli \& Schwarz 2014), and is compatible with all theories positing an extra mechanism responsible for suspension.

With respect to the relationship between EXISTENTIAL and UNIVERS AL readings, we do not yet have empirical grounds for determining whether one is more basic, which would in turn inform the choice between theories. In order to distinguish between the theoretical options, we will turn next to a second experiment, testing the same sentences in preschool-aged children. Comparing adults' and children's behavior often provides insights into how different interpretations relate to one another. In our case, children's interpretations of the sentences above could help us to identify which reading of a negatively quantified sentence is basic and which is derived. This rationale relies on a common assumption about the link between theories and acquisition: when a sentence is associated with more than one reading and children exhibit only a subset of the readings that are available to the adult, this may suggest that the latter readings are easier to access in some important way. One possibility is that readings that are harder to access are derived through additional steps or mechanisms that are either not yet acquired or difficult to master at the relevant stage of development. Granting this assumption, we can use the comparison between children and adults to further investigate the relationship between the different readings of a sentence. In this case, we can test whether one of the EXISTENTIAL or UNIVERSAL readings is basic.

\subsection{Experiment 2}

Methods and design The key elements of the experimental design were the same as in the adult version, with some minor changes in the procedure (outlined below).

Participants \& procedures Twenty-two children $(4 ; 00-5 ; 10, M=5 ; 04)$ participated in the experiment. These children were recruited from an online participant database administered by Macquarie University. Children were for the most part presented with the same experiment as that described in Experiment 1, except that they were tested in the lab at Macquarie University rather than online, and the test sentences were presented live by a researcher rather than as pre-recorded audioclips. These minimal differences between Experiment 1 and Experiment 2 were necessary to ensure that the task was appropriate for children in the target age range. We also chose not to collect response times from the child participants. 


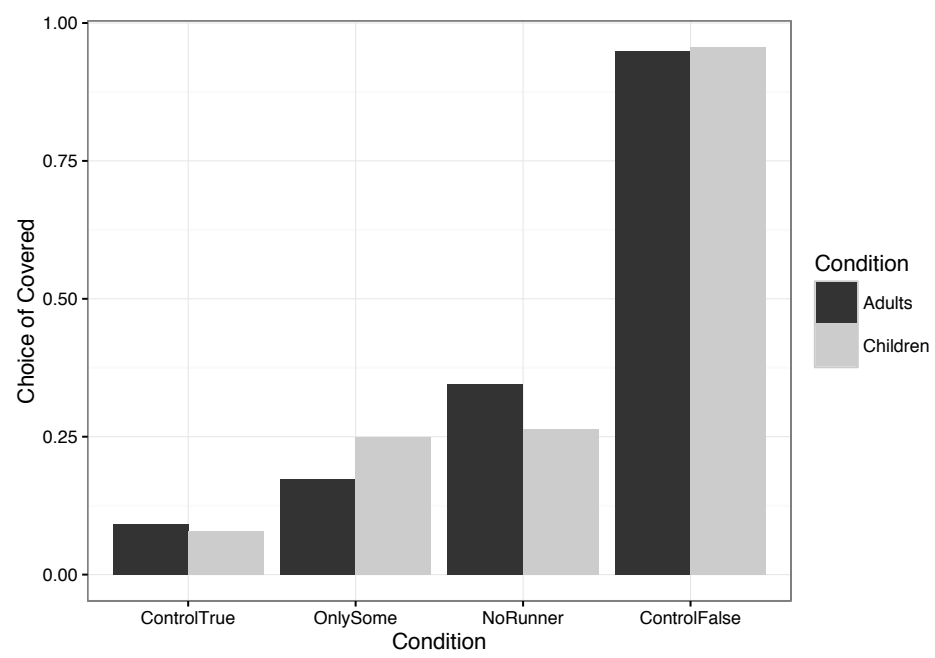

Figure 8 Proportion of covered picture selections in each condition, for adults and children. The TrueControl and FalseControl bars include the responses to both the control and the filler trials.

Predictions Assuming that children tend to prefer basic over derived meanings of sentences, the universal-only and existential-only theories predict that children should only display UNIVERSAL or EXISTENTIAL readings, respectively. In contrast, theories that include both universal and existential projection predict that children should display both UNIVERSAL and EXISTENTIAL readings.

Results As with the adult data in Experiment 1, we collapsed the control and filler trials. Two children were excluded from analyses because they scored less than $75 \%$ accuracy on these control trials. Figure 8 plots the proportions of covered picture choices by condition for the 20 child participants alongside the adult rates. We fitted mixed-effect logistic regression models to the child responses alone as well as with the inclusion of the adult responses from Experiment 1; modeling the adult and child data together or separately did not yield any qualitative differences in the observed patterns.

Focusing on the child data, the rates of covered responses in the OnlySome and in the NoRunner conditions did not differ significantly $(p=0.84)$. The two conditions both differed significantly, however, from the TrueControl condition (both $p<.001$ ). A comparison of the child and adult responses revealed no significant differences in the critical conditions ( $p=0.29$ in OnlySome and $p=0.24$ in NoRunner), but did yield a significant interaction between Group and critical conditions $(p<.05)$. 
Discussion Experiment 2 aimed to provide insight into whether universal or existential projection was the basic type of projection, based on the logic that children might be less likely than adults to access derived rather than basic meanings. Our results suggest that children have access to the UNIVERSAL reading, as acceptance of the visible picture was less frequent in the OnlySome than in the TrueControl condition. The results do not provide direct evidence, however, for children's access to the EXISTENTIAL reading, since the NoRunner condition yielded as much acceptance of the visible picture as the OnlySome condition did. In light of our reasoning above, this supports Universal-Only theories of projection. This does not preclude existential projection theories from accounting for the results in other ways; however, such theories need to provide an explanation for why children do not appear to access the EXISTENTIAL reading, even though they assume it to be basic.

\section{General Discussion}

In this study, we set out to shed light on a number of unresolved questions related to presupposition projection from the scope of negative quantifiers. First, we wanted to see if we could find empirical support for all three of the readings assumed by different accounts in the literature. Specifically, we aimed to be able to detect evidence for the EXISTENTIAL, UNIVERSAL, and PRESUPPOSITIONLESS readings of sentences like (3). Experiment 1 was designed to test for the presence of these readings in adults. The behavioral response results from this experiment suggest that adults were indeed accessing all three readings to some extent. We take these results to indicate an affirmative answer to our first research question, providing empirical support for the existence of all three readings.

The second research question our study sought to investigate was how the available readings were related to each other, and, for instance, whether one reading might be the result of the basic projection pattern, and the other derived through an additional mechanism. To this end, we ran the experiment with child participants. The reasoning behind Experiment 2 was that if children only accessed one of the relevant readings, it was likely that this reading was directly produced via projection, whereas the other reading might be derived through some additional weakening or strengthening mechanism that children had yet to acquire or master. Children's behavior in Experiment 2 suggested that they were only accessing UNIVERSAL readings. This, in turn, suggests an answer to our second research question, namely that the UNIVERSAL reading is the result of basic projection, and that the EXISTENTIAL reading, available only to adults, is derived through the application of some additional weakening mechanism, presumably domain restriction. In one follow-up experiment, we attempted to further test the idea of weakening by domain restriction, by explicitly fixing the domain of quantification. We added an overt numeral to the 
Zehr, Bill, Tieu, Romoli and Schwarz

test sentences (e.g., (12)), and ran the experiment with a new group of adults.

(12) None of the three bears won the afternoon race.

Interestingly, there was no change in behavioral patterns, compared to Experiment 1; participants still accessed the EXISTENTIAL reading. This result reduces the plausibility of domain restriction being the relevant 'weakening' mechanism to some extent. However, it may also be that adults can override the explicit numeral and proceed with domain restriction anyway, based on the results of Geurts \& van Tiel (2015). Thanks to Danny Fox (p.c.) for discussion of this point.

The third and final question our study was designed to investigate was that of the nature of PRESUPPOSITIONLESS readings. Specifically, we wanted to shed further light on the question of whether PRESUPPOSITIONLESS readings are a result of the presupposition never having been obtained at all, or if the presupposition is obtained first, and is subsequently suspended, through some additional step. The response time results from Experiment 1 showed a slow-down in adult responses that were based on the PRESUPPOSITIONLESS reading. This seemed to suggest that the PRESUPPOSITIONLESS reading is accessed through some additional step (e.g., local accommodation). However, the results from Experiment 2 complicate this picture, especially when compared to previous results from a study by Bill, Romoli, Schwarz \& Crain (2016). Bill et al. (2016) also investigated whether adults and children could access PRESUPPOSITIONLESS readings, by looking at simple negative sentences like (13) to see if participants always included the (projected) presupposition (14) in the meaning. Bill et al. (2016) found that while adults quite readily accessed a PRESUPPOSITIONLESS reading of (13), children were much less likely to do so. These results were taken by the authors as evidence that children were generally either less likely or less able to carry out the additional mechanism required to access the PRESUPPOSITIONLESS reading of (13).

(13) Bear didn't win the race.

(14) Bear participated in the race.

If we accept this analysis of Bill et al. (2016)'s results, then it is surprising that the children in our Experiment 2 accessed PRESUPPOSITIONLESS readings. The result is made even more surprising given our sentences were ostensibly more complex than those presented in Bill et al. (2016). If the PRESUPPOSITIONLESS reading is accessed through some additional step, one might have expected to observe lower rates of the reading. One possible way to reconcile Bill et al. (2016)'s results with those of our Experiment 2 is to suggest that there might be two different routes to the PRESUPPOSITIONLESS reading. One route (and perhaps the predominant one for adults) could be the application of an additional step involving suspension 
of the presupposition, as described above. Alternatively, it might be that in the face of highly complex sentences such as those we investigated, the computation of the presupposition is simply abandoned and only the literal truth conditions of the sentence are computed. The idea that presuppositions and literal truth conditions can be processed separately fits well with a two-dimensional view of meaning (Karttunen \& Peters 1979; Sudo 2012; Mandelkern 2016). That is, the PRESUPPOSITIONLESS reading is accessed not through the application of an additional step, but rather as the result of an incomplete meaning computation, due to the demands of processing such complex sentences. This kind of proposal might explain why children were apparently unable to access the PRESUPPOSITIONLESS reading of sentences like (13), while accessing such a reading from the more complex sentences investigated in the present study. According to this analysis, the answer to our third question would be that there are two ways to access the PRESUPPOSITIONLESS reading, only one of which requires the application of an additional step such as local accommodation.

\section{Conclusion \& outlook}

This study investigated presupposition projection from the scope of negative quantifiers. In particular, we focused on identifying the different readings that are possible for such sentences, and the processes through which they come about. The adult data from Experiment 1 provide empirical support for the existence of all three of the candidate readings of our test sentences (EXISTENTIAL, UNIVERSAL, and PRESUPPOSITIONLESS). The child data from Experiment 2 suggest that the UNIVERSAL reading arises from the basic projection mechanism, with the EXISTENTIAL reading being derived through some additional mechanism such as domain restriction. Reaction time data from adults reveal a delay associated with the PRESUPPOSITIONLESS reading, suggesting that it involves an additional costly mechanism (e.g., local accommodation), in line with previous results in the literature (Chemla \& Bott 2013; Romoli \& Schwarz 2014). In addition, the child data, combined with previous results from Bill et al. (2016), suggest that in the case of negative quantifiers, there may be two routes for arriving at the PRESUPPOSITIONLESS reading.

Future research could further explore and test these ideas, for example, by investigating other presupposition triggers and other quantifiers to see if the current results generalize. In particular, investigating a different quantifier like the existential some (e.g., Some of the bears won the race) would allow us to further explore the hypothesis that children only access UNIVERSAL readings - in a domain where adults tend to predominantly access EXISTENTIAL ones (Chemla 2009b; Sudo et al. 2012). Future work could also further investigate our suggestion that increased sentence complexity may be encouraging children to access the PRESUPPOSITIONLESS reading. One could accomplish this by varying sentence complexity, and measuring the 
effect on the rate of PRESUPPOSITIONLESS readings accessed by children, perhaps in comparison to adults.

\section{References}

Barr, Dale J., Roger Levy, Christoph Scheepers \& Harry J. Tily. 2013. Random effects structure for confirmatory hypothesis testing: Keep it maximal. Journal of Memory and Language 68(3). 255-278. doi:10.1016/j.jml.2012.11.001.

Beaver, David. 1994. When variables don't vary enough. In Mandy Harvey \& Lynn Santelmann (eds.), Semantics and Linguistic Theory (SALT) 4, 35-60. Ithaca, NY: CLC Publications.

Bill, Cory, Jacopo Romoli, Florian Schwarz \& Stephen Crain. 2016. Scalar implicatures versus presuppositions: The view from acquisition. Topoi 35(1). 57-71.

Charlow, Simon. 2009. "Strong" predicative presuppositional objects. In Thomas Icard (ed.), European Summer School in Logic, Language and Information ESSLLI 2009, Bordeaux.

Chemla, Emmanuel. 2009a. Presuppositions of quantified sentences: Experimental data. Natural Language Semantics 17(4). 299-340.

Chemla, Emmanuel. 2009b. Similarity: Towards a unified account of scalar implicatures, free choice permission and presupposition projection. Under revision for Semantics and Pragmatics .

Chemla, Emmanuel. 2010. Similarity: Towards a unified account of scalar implicatures, free choice permission and presupposition projection. Unpublished manuscript.

Chemla, Emmanuel \& Lewis Bott. 2013. Processing presuppositions: Dynamic semantics vs pragmatic enrichment. Language and Cognitive Processes 38(3). 241-260.

Chemla, Emmanuel \& Philippe Schlenker. 2012. Incremental vs symmetric accounts of presupposition projection: An experimental approach. Natural Language Semantics 20(2). 177-226.

Chierchia, Gennaro. 1995. Dynamics of Meaning. Chicago: University of Chicago Press.

Chierchia, Gennaro. 2010. NPIs. Unpublished manuscript.

Fox, Danny. 2008. Two short notes on schlenker's theory of presupposition projection. Theoretical Linguistics 34(3). 237-252.

Fox, Danny. 2011. Lecture notes. Pragmatics Class, MIT.

Fox, Danny. 2012. Cancelling the maxim of quantity: Another argument for a grammatical derivation of scalar implicatures. Unpublished manuscript MIT/HUJI. 
Presupposition projection from the scope of none

George, Benjamin. 2008. Presupposition Repairs: A Static, Trivalent Approach to Predicting Projection. UCLA MA thesis.

Geurts, Bart. 1998. Presuppositions and anaphors in attitude contexts. Linguistic and Philosophy .

Geurts, Bart \& Bob van Tiel. 2015. When "all the five circles" are four: New exercises in domain restriction. Topoi [Advance Access]. doi:10.1007/s11245014-9293-0.

Heim, Irene. 1983. On the projection problem for presuppositions. In Daniel P. Flickinger (ed.), West Coast Conference on Formal Linguistics (WCCFL) 2, 114-125. Stanford University, Stanford, California: CSLI Publications.

Huang, Yi Ting, Elizabeth Spelke \& Jesse Snedeker. 2013. What exactly do numbers mean? Language Learning and Development 9(2). 105-129.

Karttunen, Lauri. 1974. Presupposition and linguistic context. Theoretical Linguistics 1. 181-194.

Karttunen, Lauri \& Stanley Peters. 1979. Conventional implicature. Syntax and Semantics 11. 1-56.

Mandelkern, Matthew. 2016. Dissatisfaction theory. In Mary Moroney, Carol-Rose Little, Jacob Collard, and Dan Burgdorf (ed.), Semantics and Linguistic Theory (SALT) 26, 391-416. Ithaca, NY: CLC.

Mayr, Clemens \& Uli Sauerland. 2016. Accommodation and the strongest meaning hypothesis. In Thomas Brochhagen, Floris Roelofsen and Nadine Theiler (ed.), Amsterdam Colloquium 2015, 276-285.

Romoli, Jacopo. 2012. Soft but strong: Neg-raising, soft triggers, and exhaustification. Harvard University PhD dissertation.

Romoli, Jacopo. 2014. The presuppositions of soft triggers are obligatory scalar implicatures. Journal of Semantics 32(2). 173-219.

Romoli, Jacopo \& Florian Schwarz. 2014. An experimental comparison between presuppositions and indirect scalar implicatures. In Florian Schwarz (ed.), Experimental Perspectives on Presuppositions. Studies in Theoretical Psycholinguistics, 215-240. Dordrecht: Springer.

Schlenker, Philippe. 2008. Presupposition projection: The new debate, 655-693.

Schlenker, Philippe. 2010. Local contexts and local meanings. Philosophical Studies 151(1). 115-142. doi:10.1007/s11098-010-9586-0.

Stalnaker, Robert. 1970. Pragmatics. Synthese 22. 272-289.

Stalnaker, Robert. 1974. Pragmatic presuppositions. In M. Munitz \& D. Unger (eds.), Semantics and Philosophy, 197-213. New York University Press.

Sudo, Yasutada. 2012. On the semantics of phi features on pronouns. Cambridge, MA Massachusetts Institute of Technology $\mathrm{PhD}$ thesis.

Sudo, Yasutada, Jacopo Romoli, Danny Fox \& Martin Hackl. 2012. Variation of presupposition projection in quantified sentences. In Floris Roelofsen Galit 
Weidman Sassoon Katrin Schulz Maria Aloni, Vadim Kimmelman \& Matthijs Westera (eds.), Amsterdam Colloquium 2011, Amsterdam, The Netherlands. van der Sandt, Rob. 1992. Presupposition projection as anaphora resolution. Journal of Semantics 9. 333-377.

Jérémy Zehr

619 Williams Hall

University of Pennsylvania

Philadelphia, PA 19104

jeremy.e.zehr@gmail.com

Lyn Tieu

Department of Cognitive Science

16 University Avenue

Macquarie University

NSW 2109 Australia

lyn.tieu@gmail.com

Florian Schwarz

619 Williams Hall

University of Pennsylvania

Philadelphia, PA 19104

florians@sas.upenn.edu
Cory Bill

Department of Cognitive Science

16 University Avenue

Macquarie University

NSW 2109 Australia

corybill@gmail.com

Jacopo Romoli

Shore Road

Ulster University

Newtownabbey, UK, BT37 0QB

j.romoli@ulster.ac.uk 